\title{
LA COOPERACIÓN INTERNACIONAL EN LA LUCHA CONTRA EL TERRORISMO YIHADISTA EN EL SENO DE LA UNIÓN EUROPEA TRAS LOS ATENTADOS TERRORISTAS A PARTIR DEL AÑO 2015
}

Maitena Sol Springli y Pamela Curvale*

\section{Resumen}

A lo largo de su historia, la Unión Europea se ha enfrentado a diferentes desafíos en materia de seguridad, siendo uno de ellos el terrorismo, lo que ha colocado a la prevención del terrorismo en un lugar prioritario en la agenda del bloque.

A partir de la aprobación, en noviembre de 2005, de la primera estrategia antiterrorista de la Unión Europea, la evolución de este fenómeno y sus consecuentes atentados han llevado a las autoridades del viejo continente a realizar una adaptación periódica de sus políticas y planes para hacer frente a su amenaza.

No obstante, con el surgimiento del Estado Islámico a fines de 2014 y el impacto de sus atentados, se encara a un nuevo tipo de terrorismo yihadista, diferente de Al Qaeda, planteando un renovado escenario post Guerra Fría, tanto para el sistema internacional como para los Estados del bloque europeo. Esto lleva a preguntarse si dichos actos cometidos por una minoría fanatizada obligan a alterar las políticas en materia de seguridad de los Estados afectados, y a su vez, a reconocer la necesidad de adoptar mecanismos de cooperación dentro del bloque europeo para hacer frente a la lucha contra el terrorismo.

Palabras Clave: Unión Europea- Seguridad- Defensa- Terrorismo- Cooperación entre Estados.

\footnotetext{
* Maitena Sol Springli: Licenciada en Relaciones Internacionales, Universidad Católica Argentina, Promotora en los Objetivos del Desarrollo Sostenible, Senado de la provincia de Buenos Aires. Actualmente cursando postgrado en Cooperación Internacional. Universidad Católica de Córdoba. Miembro del CIIVIDS (Centro de Investigación Interdisciplinar en Valores, Integración y Desarrollo Social) - Facultad "Teresa de Ávila" - UCA. Contacto: maitenaspringli@uca.edu.ar. ORCID: https://orcid.org/0000-0001-9911-5446 - Pamela Curvale: Licenciada en Relaciones Internacionales, Licenciada en Ciencias Políticas - Universidad Católica Argentina, Magister en Integración y Cooperación Internacional (CERIR-CEI-UNR). Docente Universidad Católica Argentina sede Paraná. Miembro del CIIVIDS (Centro de Investigación Interdisciplinar en Valores, Integración y Desarrollo Social) - Facultad "Teresa de Ávila" - UCA. Contacto: pamelacurvale@uca.edu.ar. ORCID: https://orcid.org/0000-0001-6733-4994
} 


\title{
INTERNATIONAL COOPERATION IN THE FIGHT AGAINST JIHADIST TERRORISM WITHIN THE EUROPEAN UNION FOLLOWING THE TERRORIST ATTACKS FROM 2015 ONWARDS
}

\begin{abstract}
Throughout its history, the European Union has faced various security challenges, one of them being terrorism, which has placed terrorism prevention at the top of the bloc's agenda.

Since the approval, in November 2005, of the European Union's first antiterrorism strategy, the evolution of this phenomenon and its consequent attacks have led the authorities of the old continent to periodically adapt their policies and plans to deal with the threat of terrorism.

However, with the emergence of the Islamic State at the end of 2014 and the impact of its attacks, a new type of jihadist terrorism, different from Al Qaeda, is being faced, posing a renewed post-Cold War scenario, both for the international system and for the states of the European bloc. This raises the question of whether such acts committed by a fanatical minority force a change in the security policies of the States concerned and, in turn, recognize the need to adopt cooperation mechanisms within the European bloc to deal with the fight against terrorism.
\end{abstract}

Key Words: European Union- Security- Defense- Terrorism- Cooperation between states.

TRABAJO RECIBIDO: 30/03/2021 - TRABAJO ACEPTADO: 26/05/2021 


\section{Introducción}

A partir del impacto generado por el surgimiento de nuevos actores internacionales y el desarrollo de una agenda con mayor amplitud sobre los asuntos globales, el escenario internacional se ha tornado más complejo dando forma a mecanismos de cooperación basados en renovados pilares y en la implementación de acciones conjuntas que permitan paliar los desafíos y amenazas globales y regionales.

En relación a lo mencionado, la Unión Europea (en adelante UE) se ha visto comprometida a lo largo de las últimas décadas por diversas amenazas, sorteando crisis de diferente gravedad e impacto, como lo es el terrorismo.

La importancia de analizar el fenómeno terrorista en la sociedad internacional atiende a varias razones, siendo una de ellas la modificación sufrida por las agendas internacionales de seguridad, al ubicar al terrorismo como una de sus principales amenazas en la última década, con el consecuente diseño, implementación y seguimiento de medidas antiterroristas (Springli, 2019).

En lo concerniente a Europa, el terrorismo se ha convertido en un reto para los Estados, que han observado el desarrollo de este fenómeno a través de los años. Los atentados del 11 de septiembre de 2001 (en adelante 11-S) en las torres del World Trade Center en los Estados Unidos, no fueron los primeros actos terroristas conocidos por el mundo, pero sí los primeros en cambiarlo. Después de dichos atentados, salieron a la luz las debilidades y los vacíos jurídicos, de inteligencia y de seguridad que las principales potencias tenían sobre el tema. Pocos años después de los atentados ocurridos en Estados Unidos, España e Inglaterra fueron víctimas de nuevos ataques en 2004 y 2005, respectivamente. Laurence Thieux (2004) explica que estos atentados suscitaron una reacción inmediata por parte de los responsables de las instituciones europeas, las cuales reafirmaron su voluntad de dar una respuesta común y coordinada a una amenaza de seguridad interna a la cual los Estados por sí solos difícilmente podrían enfrentarse.

Para afrontar esta situación, desde la UE se decidió adoptar una serie de medidas, siendo una de las más importantes la creación de la primera estrategia antiterrorista en 2005, con el objetivo de abordar la prevención y represión del terrorismo en sus diversas manifestaciones, impulsando la respuesta regional ante un fenómeno que compromete la seguridad de la ciudadanía y de los Estados.

Es a partir del año 2015 que surge la necesidad dentro del bloque europeo, de reforzar la política de seguridad frente al terrorismo, como consecuencia de la aparición en escena del Estado Islámico (en adelante EI), un nuevo grupo terrorista que ha perpetrado diversos atentados en el continente europeo entre los que se pueden mencionar los 
atentados de París ${ }^{1}$ en noviembre de 2015, los ocurridos en Bruselas ${ }^{2}$ en marzo de 2016, en $\mathrm{Niza}^{3}$ en julio del mismo año o en Londres ${ }^{4}$ en marzo de 2017 (GRESH, 2015). Este grupo, y su efecto en la dinámica terrorista, se ha convertido en los últimos tiempos en una de las problemáticas que plantean un renovado escenario post Guerra Fría para el sistema internacional y para los Estados del bloque europeo, llevando a preguntarse si actos cometidos por una minoría fanatizada obligan a alterar las políticas de seguridad para el bloque europeo y a su vez, a reconocer la necesidad de un trabajo en conjunto para hacer frente a la lucha contra el terrorismo (Springli, 2019).

Es a partir de este escenario, que el presente artículo tiene como objetivo principal describir los mecanismos de cooperación llevados a cabo en el seno de la UE respecto a la lucha contra el terrorismo, tras el surgimiento del EI y la serie de atentados terroristas mencionados, a partir del año 2015.

En cuanto a la metodología, este trabajo presenta un carácter cualitativo, con un diseño exploratorio - descriptivo. Avanzando con la función operativa y con la finalidad de proveer de mayor claridad, se presentan a continuación las diferentes técnicas de recolección de datos utilizadas:

- Análisis de material documental: representa la principal técnica de recolección de datos para poder interpretar el corpus empírico. Esta se efectuó mediante la recolección y el análisis de:

- Legislaciones: conjunto de documentos oficiales (leyes, decretos, declaraciones y tratados) emitidos por la UE respecto a la lucha contra el terrorismo. Paralelamente, se realizó la búsqueda de material documental pertinente a la temática, a través de portales oficiales de la organización como el del Consejo Europeo y el Consejo de la UE.

\footnotetext{
${ }^{1}$ Los atentados en París de noviembre de 2015 fueron varios ataques terroristas cometidos en la noche del 13 de noviembre en París y su suburbio de Saint-Denis, perpetrados por atacantes suicidas islamistas en los que murieron 131 personas y otras 415 resultaron heridas. Los ataques consistieron en tiroteos contra terrazas de 5 bares y restaurantes, tiroteo y toma de rehenes en la sala de conciertos Bataclan y en explosiones suicidas alrededor del Estadio de Francia y en otro restaurante cerca de la Plaza de la Nación. La autoría de los ataques fue reivindicada por el El. Es considerada la peor masacre ocurrida en territorio continental francés desde el final de la Segunda Guerra Mundial.

2 Los atentados de Bruselas de 2016 fueron dos ataques terroristas realizados por seguidores del autoproclamado El la mañana del martes 22 de marzo de 2016 en el aeropuerto y la red de metro de la capital belga en los que murieron 35 personas (incluyendo tres de los terroristas) y 340 resultaron heridas.

${ }^{3}$ El atentado de Niza de 2016 fue un ataque terrorista que tuvo lugar en la ciudad francesa de Niza el 14 de julio de 2016, cuando Mohamed Lahouaiej Bouhlel, un residente tunecino en Francia afiliado al El, condujo deliberadamente un camión de carga de 19 toneladas hacia una multitud que estaba celebrando el Día Nacional de Francia, en el paseo de los Ingleses, matando a 86 personas e hiriendo a 434.

${ }^{4}$ El atentado de Londres de 2017 ocurrió el sábado 3 de junio en el Puente de Londres y en Borough Market, Londres (Reino Unido), donde un grupo de tres terroristas pertenecientes al El, condujo un vehículo por la acera del Puente de Londres, atropellando a los viandantes.
}

Esta obra se encuentra bajo la Licencia Creative Commons Reconocimiento - Compartir Igual (by-sa) 
- Notas de prensa e información digital: se analizaron las notas de prensa relacionadas a la temática a estudiar, así como información digital obtenida de portales informativos como: France 24, BBC Mundo, y Europa Press. Así mismo, se utilizaron fuentes de portales oficiales de la UE como: web oficial de la Comisión Europea, web oficial del Parlamento Europeo y web oficial de la UE.

- Publicaciones: se analizaron informes elaborados por la UE respecto al terrorismo extraídos de la página oficial del Consejo Europeo y del Consejo de la UE respecto a la lucha contra el terrorismo.

- Entrevista semiestructurada: la misma fue realizada a un informante clave, funcionario público que, si bien desempeña sus funciones dentro del gobierno francés y las áreas específicas del Ministerio para Europa y de Asuntos Exteriores, ha jugado y juega un rol destacado en la gestión respecto a la seguridad y defensa frente al terrorismo de toda la región europea. Por medio de una guía de contenido con preguntas abiertas, se entrevistó al Jefe de Gendarmería Coronel Joel Molera, actualmente desempeñando su cargo en la Embajada Francesa en Argentina.

Teniendo en cuenta lo mencionado, se presentan tres apartados que desarrollan los contenidos para responder al objetivo propuesto: el apartado 1, introduce el concepto de cooperación y explica cómo se constituye en uno de los objetivos de política de seguridad en el seno de la UE. En el apartado 2 se avanza en describir el proceso de la reaparición del terrorismo yihadista como amenaza en el escenario europeo y, en complemento, se abordan las características del fenómeno terrorista y la evolución de uno de los grupos más conocidos en la actualidad, el EI. Por último, en el apartado 3, se analiza cuál ha sido la respuesta al terrorismo desde el seno de la UE a partir de dos subapartados. Por un lado, en el subapartado 3.1, se describen los antecedentes de cooperación en materia de lucha contra el terrorismo previo a los atentados terroristas del año $2015 \mathrm{y}$, por el otro, en el subapartado 3.2 se describe cómo se ha ido renovando la Estrategia de Seguridad Europea a partir de los atentados de 2015.

\section{La cooperación como objetivo en la política de seguridad en el seno de la Unión Europea}

Como se mencionó, las transformaciones del escenario internacional a partir del surgimiento de nuevos actores que se relacionan en un mundo más interdependiente, junto con una agenda más amplia que contempla nuevos temas globales, han impulsado la creación de nuevos mecanismos de cooperación basados en la implementación de acciones conjuntas para hacer frente a las amenazas internas, regionales e internacionales.

Existe un consenso acerca de la definición de cooperación, vinculada fundamentalmente a las ideas neoliberales de Robert Keohane, quien sostiene que la cooperación es entendida como: "un proceso a través del cual las políticas seguidas por los gobiernos llegan a ser consideradas por los asociados como acciones que facilitan sus propios objetivos, como resultado de la coordinación de políticas" (Keohane, 1988: 88). Siguiendo con esta línea, Vachino plantea que la cooperación abarca el establecimiento y 
la concertación de acuerdos y proyectos específicos, múltiples pero autónomos, fundados en el mutuo interés y conveniencia, y que finaliza cuando se cumple el fin determinado o decae el interés que lo motivó (en Mellado, 2002).

Por otro lado, la cooperación entre Estados puede ser analizada desde dos ópticas: en primer lugar, como instrumento de política exterior $y$, en segundo lugar, como objetivo de política exterior. Teniendo en cuenta estas consideraciones es posible afirmar que, en el primer caso, conforme a las ideas de Hans Morgenthau (1986), la cooperación es considerada como un instrumento de política exterior cuando busca consolidar una posición de poder y, como tal, forma parte de una decisión política. Por ello la cooperación, para la teoría realista, se presenta como un instrumento de la política exterior de los Estados. En cambio, si la cooperación es entendida como un mecanismo para alcanzar la paz, para garantizar la seguridad de los Estados y sus ciudadanos, se podría plantear la misma como un objetivo dentro la política exterior.

Los objetivos que motivan la cooperación entre Estados pueden ser muy diversos: en términos económicos, se buscaría incrementar la eficiencia y/o reducir los costos en las transacciones realizadas por los países; en términos sociales, el motor impulsor de la cooperación sería el deseo de reducir externalidades; y en términos políticos y militares, se cooperaría con el fin de protegerse frente a amenazas externas comunes, como por ejemplo el terrorismo, de acuerdo a lo analizado en el presente artículo.

Teniendo en cuenta estas motivaciones, Keohane plantea que "existen razones válidas para creer que en ciertas condiciones la reciprocidad específica puede facilitar la cooperación en la política mundial" (1993: 196). Por lo que se puede observar que existen intereses comunes que propician la cooperación a escala internacional y por esto tienden a prevalecer los beneficios para todas las partes involucradas.

Se observa que este mecanismo de cooperación permite subsanar problemáticas concretas y paliar amenazas, favoreciendo el desarrollo social y económico de los Estados y de las regiones, permitiendo consensuar espacios de estabilidad política, el predominio de la paz, la gobernabilidad y la seguridad.

En materia de integración, la cooperación internacional ha estado presente de manera constante a lo largo de la historia de las relaciones internacionales. La formación de la UE es uno de los ejemplos más conocidos, representando un proceso de integración que surge en los años 50 del siglo pasado, con el objetivo de culminar con los conflictos suscitados en Europa hasta la Segunda Guerra Mundial y así alcanzar una paz duradera manteniendo la seguridad regional por medio de medidas conjuntas y una cooperación entre los miembros.

En base a lo desarrollado y considerando las aproximaciones conceptuales, uno de los factores que a lo largo de las décadas ha guiado el accionar del bloque a cooperar y hacer frente ante las nuevas amenazas, es el terrorismo. Este fenómeno, el cual será abordado con mayor profundidad en el apartado siguiente, plantea un escenario complejo, ya que por sus características hace difícil que los Estados puedan luchar de manera individual contra el mismo. Sin embargo, siguiendo las ideas de Javier Albaladejo (2019), 
la lucha contra el terrorismo, desde el punto de vista del denominado núcleo esencial de la soberanía nacional, ha hecho especialmente difícil la cooperación en la UE en esta materia. En efecto, la responsabilidad de la lucha contra el terrorismo sigue siendo de las autoridades nacionales de cada Estado miembro. Las dudas iniciales de muchos Estados miembros a cooperar en este ámbito han ido evolucionando hasta el punto de considerar hoy en día esa cooperación como esencial e indispensable para afrontar el terrorismo, considerado como una de las amenazas más graves para la seguridad de los ciudadanos europeos.

\section{La reaparición del yihadismo como amenaza en el escenario europeo}

El fenómeno del terrorismo yihadista supone un desafío en sí mismo debido a la falta de consenso a la hora de definirlo y prevenirlo. Coincidiendo con lo sostenido por Anabella Busso (2003), podemos decir que el terrorismo no constituye una forma nueva de violencia, ya que existen datos que nos permiten rastrear numerosas experiencias terroristas a lo largo de la historia. Sin embargo, el contexto de post Guerra Fría y globalización ha favorecido su incremento $\mathrm{y}$, como consecuencia de ello, su protagonismo se ha multiplicado, dando lugar a una preocupación creciente por parte de la región europea, que ha evidenciado el surgimiento de nuevos grupos terroristas como el EI y el afianzamiento de otros ya conocidos, como Al Qaeda y sus ramas regionales (Velasco Tuduri, 2013).

Por otro lado, así como las relaciones internacionales se han ido adaptando a los cambios en el sistema internacional, el terrorismo ha ido evolucionando a lo largo de los años, encontrándonos con una amenaza que ha adquirido nuevas facetas. Laurence Thieux (2004) plantea que es necesario tener en cuenta que el fenómeno del terrorismo suele describirse a partir de su singularidad: se habla de terrorismo internacional, transnacional o global, o de terrorismo islámico. Del mismo modo, sostiene que en cuanto al accionar terrorista es importante considerar la diversidad de las situaciones políticas, económicas e ideológicas que conducen a un determinado grupo a optar por esta estrategia.

Es a raíz de lo mencionado que no existe en la actualidad una definición consensuada internacionalmente acerca del terrorismo. No obstante, el Consejo Europeo en su sesión extraordinaria del 21 de septiembre de $2001^{5}$, declaró que el terrorismo era un verdadero reto para el mundo y para Europa y que la lucha contra el terrorismo sería un objetivo prioritario de la UE.

Por otro lado, en el Artículo K.1 del Tratado de la UE (en adelante TUE), se plantea que el terrorismo debe prevenirse y combatirse desarrollando acciones comunes a partir de la colaboración estrecha entre las autoridades policiales, las autoridades aduaneras y otras autoridades competentes. Así mismo, se manifiesta la necesidad de incrementar la colaboración entre las autoridades judiciales y otras autoridades competentes de los Estados miembros; y la aproximación de las normas penales, en caso de ser necesario

\footnotetext{
${ }^{5}$ Posición Común 2001/931/PESC del Consejo, de 27 de diciembre de 2001, sobre la aplicación de medidas específicas de lucha contra el terrorismo, DO L 344 de 28.12.200.
}

Esta obra se encuentra bajo la Licencia Creative Commons Reconocimiento - Compartir Igual (by-sa) 
(Villalba Fernández, 2007). Esto evidencia que la cooperación se encuentra entre sus principales estrategias para luchar contra el terrorismo.

Años más tarde, como resultado de los esfuerzos por parte de la UE para definir al terrorismo, mediante una directiva adoptada por el Consejo de la UE en 2017, se establecieron normas mínimas relativas a la definición de las infracciones penales y las sanciones en el ámbito del terrorismo y se definió que los delitos de terrorismo son:

Aquellos actos cometidos con el objetivo de intimidar seriamente a una población, obligar indebidamente a un gobierno u organización internacional a realizar o abstenerse de realizar cualquier acto y/o desestabilizar gravemente o destruir las estructuras políticas, constitucionales, económicas o sociales fundamentales de un país o una organización internacional (Diario Oficial De La Unión Europea, 2017).

A partir de las aproximaciones desarrolladas con anterioridad, se procederá con la descripción de las principales características del EI, el cual se constituye como uno de los grupos terroristas que mayor incidencia ha tenido en Europa en los últimos años y que surge en el contexto de la ocupación de Irak en 2003. Es importante aclarar que para esto se tendrá en cuenta también el desarrollo del grupo terrorista Al Qaeda, ya que el EI surgió como una rama que se desprende del mismo.

En concordancia con lo que plantea Fernando Reinares (2011) Al Qaeda se originó en 1988, bajo el liderazgo del saudí Osama Bin Laden, como una organización paramilitar y yihadista que surgió como un movimiento de resistencia islámica alrededor de la contienda que la invasión soviética desencadenó en Afganistán y la guerra que tuvo lugar a lo largo de la década del 80 del siglo pasado. Su desenlace supuso la fundación de dicha organización inspirada en las actitudes y creencias propias del salafismo yihadista que dio lugar a la creación de un heterogéneo conjunto de organizaciones asociadas con Al Qaeda Central o sus extensiones, sumando el elenco de individuos - lobos solitarios - y células independientes, inspirados por la ideología y las directrices de la organización central como matriz del yihadismo global.

Mencionado esto, se pueden distinguir tres períodos que ha atravesado el yihadismo global:

- Primer período (1988-2001): Se inicia con la formación de Al Qaeda como núcleo funcional y matriz de referencia del terrorismo propiamente dicho. Esta etapa concluye en 2001 con los atentados del 11-S y sus inmediatas repercusiones a nivel global, dando inicio a un nuevo período.

- Segundo período (2001-2011): Esta etapa inicia con los atentados del 11$\mathrm{S}$ y es una de las más importantes para el movimiento ya que Al Qaeda, junto con sus extensiones territoriales, llevaron a cabo otros atentados de gran magnitud como los del 11 de marzo de 2004 en Madrid (11-M), el 7 de julio de 2005 en Londres (7-J), entre otros. Dicha etapa termina en 2011 con el abatimiento de Osama Bin Laden y el comienzo de las convulsiones políticas en algunos países del mundo árabe, a lo largo del cual Al Qaeda 
se descentraliza y el yihadismo global adquiere rasgos de un fenómeno polimorfo.

- Tercer período (2011-actualidad): Período actual, en el que el yihadismo global se encuentra más extendido que nunca, pero dividido ahora entre sus dos matrices de referencia: Al Qaeda y, desde junio de 2014 en adelante, el denominado EI. Es en este último período en el que se inserta el fenómeno del EI (también llamado Daesh), que se analizará a continuación.

Las raíces de EI se remontan al año 2002, cuando el fallecido Abu Musab alZarqawi creó el grupo radical Tawhidwa al-Jihad. El el 20 de marzo de 2003, un año después de la invasión a Irak a cargo de una coalición liderada por Estados Unidos junto a países aliados como Reino Unido y España, Zarqawi juró lealtad a Osama Bin Laden y fundó Al Qaeda en Irak, convirtiéndose en la mayor fuerza insurgente durante los años de la ocupación estadounidense. Sin embargo, después de la muerte de Zarqawi en 2006, Al Qaeda creó una organización alterna llamada el Estado Islámico de Irak (ISI, por sus siglas en inglés). ISI fue debilitada por las tropas de Estados Unidos y por la creación de consejos Sahwa (Despertar), liderados por tribus sunitas que rechazaron la brutalidad de ISI. En 2010 Abu Bakr al-Baghdadi se convirtió en el nuevo líder, reconstruyó la organización y realizó múltiples ataques en el país. En 2013 se unió a la rebelión contra el presidente sirio, Bashar al Asad, junto al frente al Nusra. En abril de ese año, Abu Bakr anunció la fusión de las milicias en Irak y Siria y las bautizó como Estado Islámico de Irak y el Levante (ISIS, por sus siglas en inglés). Los líderes del frente Al Nusra, con el que habían combatido contra al Asad, rechazaron la decisión. Pero los combatientes leales a Abu Bakr lo siguieron en su empeño yihadista.

En diciembre de 2013, ISIS se enfocó en Irak y aprovechó la profunda división política entre el gobierno de orientación chiíta y la minoría sunita. Ayudados por los líderes tribales, lograron controlar la ciudad de Faluya. Pero el verdadero golpe se efectuó en junio de 2014, cuando tomaron el control de Mosul, la segunda ciudad del país y continuaron su avance hacia la capital, Bagdad. A mediados de julio habían consolidado su control sobre docenas de ciudades y localidades. En ese punto, ISIS declaró la creación del Califato ${ }^{6}$ y cambió su nombre al de Estado Islámico constituyéndose como "un grupo insurgente con una destacable capacidad paramilitar, superior a la que había ostentado en los mejores momentos de la década pasada" (Jordán, 2013: 123), aprovechando la oportunidad política de la grave inestabilidad producto de la invasión estadounidense a Irak y el descontento social provocado por la invasión de algunas potencias occidentales, lo que potenció la sensación de resentimiento y frustración con respecto de las mismas, otorgándole cada vez más legitimidad a las ideas radicalizadas del yihadismo.

El EI, que comenzó siendo un grupo terrorista de origen interno al Estado iraquí respondiendo a la invasión norteamericana, pasó a ser internacional al expandir sus actividades a Siria y luego al resto de la región, con intenciones de reinstaurar el dominio

\footnotetext{
${ }^{6}$ Un califato es una forma de Estado dirigido por un líder político y religioso de acuerdo con la ley islámica o sharia.
} 
político de la autoridad religiosa musulmán de tipo sunnita en todas las tierras consideradas históricamente musulmanas, denominado Califato, reemplazando a la autoridad existente y utilizando la violencia como recurso preferente.

Para lograr comprender el impacto del EI como nuevo grupo terrorista se plantea que el mismo modificó al movimiento terrorista internacional de dos formas: por un lado, representó el surgimiento de un nuevo tipo de amenaza, ya que no se trata de un grupo terrorista convencional, sino que es una organización híbrida terrorista insurgente con aspiraciones estatales; y, por el otro, dividió la corriente yihadista en dos facciones: Al Qaeda y sus filiales bajo el liderazgo de Aymán Al-Zawahiri y el EI con su creciente número de afiliados leales a Abu Bakr Al-Baghdadi.

\section{Respuesta al terrorismo en el seno de la Unión Europea}

Considerando el accionar de los grupos terroristas que mayor incidencia han tenido en Europa en los últimos años, la UE ha decidido responder a través de diversos mecanismos de cooperación, los cuales serán detallados a continuación, haciendo una distinción entre las acciones implementadas de forma conjunta anteriores al año 2015 y posteriores a esta fecha.

\subsection{Antecedentes de cooperación entre los miembros previos a los atentados terroristas de 2015}

Las acciones europeas contra el terrorismo internacional se iniciaron en los años setenta del pasado siglo, pero sólo a partir de los años noventa, tras la aprobación del TUE, se empezaron a crear los instrumentos necesarios para avanzar en una estrategia antiterrorista común. Sin embargo, la seguridad de la UE y de sus Estados miembros ha adquirido un carácter más perentorio concretamente a raíz de los ataques terroristas que tuvieron lugar el 11-S en Estados Unidos ${ }^{7}$.

Paralelamente, mientras la UE se proponía mejorar la seguridad y avanzar en su espacio de seguridad interior y justicia, el 11 de marzo de 2004 se produjo el mayor atentado terrorista de la historia de España ${ }^{8}$ y el más grave de los cometidos en Europa por radicales islamistas ligados a $\mathrm{Al}$ Qaeda. Un año después, la tragedia terrorista se repetía en Londres el 7 de julio de $2005^{9}$, llevando a incrementar aún más la presión para acelerar la puesta en marcha de varias medidas que avanzaban lentamente en el ámbito europeo.

Para hacer frente a esta amenaza la UE decidió reasegurar los mecanismos ya adoptados en 2001, entre los que se destacó la adopción por parte del Consejo Europeo de

\footnotetext{
7 Cronología: lucha antiterrorista de la UE. Recuperado de: https://www.consilium.europa.eu/es/policies/fight-against-terrorism/history-fight-against-terrorism/

${ }^{8}$ Serie de ataques terroristas en cuatro trenes de la red de Cercanías de Madrid llevados a cabo por Al Qaeda y el Grupo Islámico Combatiente Marroquí. Fallecieron 193 personas y alrededor de dos mil resultaron heridas.

${ }^{9}$ Los atentados del 7 de julio de 2005 marcaron un antes y un después en el Reino Unido. Al día de hoy siguen siendo los ataques terroristas más sangrientos cometidos en suelo británico. Murieron 52 y más de setecientas resultaron heridas por las bombas en la red pública de transportes.
}

Esta obra se encuentra bajo la Licencia Creative Commons Reconocimiento - Compartir Igual (by-sa) 
la Estrategia Europea de Seguridad (EES) en diciembre de 2003. En ella se establecieron por primera vez principios y objetivos claros para promover los intereses de la UE en materia de seguridad, y se incluyó al terrorismo como una amenaza fundamental. Con ello, la UE evidenció, desde un punto de vista político, su voluntad de superar una dimensión puramente estatal de las cuestiones de seguridad, abordando de forma conjunta el proceso de sistematización y jerarquización de las amenazas y riesgos a los que se enfrentan los Estados de la Unión. Se trataba, en suma, de un ejercicio previo fundamental para proceder, a continuación, a la regulación jurídica de los instrumentos de respuesta imprescindibles para quien aspira a convertirse en actor global relevante en un mundo.

Así mismo, a raíz de los atentados terroristas del 11-M en 2004, los dirigentes de la UE adoptaron una declaración sobre la lucha contra el terrorismo. Entre otras medidas, acordaron crear el cargo de coordinador de la UE para la lucha contra el terrorismo, ocupando el cargo Gijs de Vries y años más tarde en 2007 fue sucedido por Gilles de Kerchove, quien continúa ejerciendo sus funciones hasta la actualidad. Entre sus funciones se destacan: coordinar la labor del Consejo de la UE en la lucha antiterrorista, supervisar la aplicación de la estrategia de la UE de Lucha contra el Terrorismo y velar por que la UE participe activamente en la lucha antiterrorista. Así mismo, el Coordinador tiene la función de celebrar reuniones regularmente con funcionarios gubernamentales y partes interesadas ajenas a la UE para intercambiar puntos de vista sobre la cooperación antiterrorista en permanente cambio.

Un año más tarde, en 2005, se implementó la primera estrategia antiterrorista, con el objeto de combatir el terrorismo a escala mundial y garantizar la seguridad en Europa. Dicha estrategia se basó en cuatro pilares fundamentales: prevenir, proteger, perseguir y responder, los cuales constituyen una respuesta global y proporcionada a la amenaza terrorista internacional. Prevenir para que las personas de religión musulmana, en especial los jóvenes, no se sientan atraídos por el terrorismo. Proteger las fronteras exteriores y los medios de transporte transfronterizos desarrollando FRONTEX, estableciendo el Sistema de Información de Visados y la segunda generación del Sistema de Información Schengen. Perseguir a los terroristas coordinadamente en todos los países de la UE y utilizando los análisis del Sit Cen (Situation Center) para desarrollar una comprensión común de la amenaza que permita desarrollar políticas comunes para responder a la misma. Y, finalmente, aceptando que no se puede reducir la amenaza a cero, responder a los posibles ataques de forma conjunta, con acuerdos de cooperación en caso de crisis y reforzando el mecanismo comunitario de protección civil (Aixalá, 2007).

Para lograr esta estrategia y cumplir con los cuatro pilares, se decidió profundizar en diversas acciones de cooperación relacionadas a la seguridad, principalmente en la cooperación judicial y policial en materia penal y en la implantación del principio de reconocimiento mutuo, que ya se venían desarrollando anteriormente a la implementación de la estrategia en 2005. En cuanto a la cooperación policial, ya en 1996 se había aprobado la creación y mantenimiento de un directorio de competencias, técnicas y conocimientos antiterroristas especializados para facilitar la cooperación antiterrorista entre los Estados miembros del bloque. Dos años más tarde, se creaba en 1998 la Oficina Europea de Policía (Europol), siendo el órgano encargado de facilitar las operaciones de lucha contra la delincuencia en el seno de la UE. Asimismo, la Decisión del Consejo con 
fecha del 3 de diciembre de 1998, encomendaba a Europol la lucha contra los delitos cometidos o que puedan cometerse en el marco de actividades terroristas que atenten contra la vida, la integridad física, la libertad o los bienes de las personas (Villalba Fernández, 2007).

Por otro lado, en materia de cooperación judicial, se encontraba como antecedente la facilitación de la extradición entre los Estados miembros, y la incorporación de dos instrumentos jurídicos primordiales: el Convenio Relativo al Procedimiento Simplificado de Extradición entre los Estados miembros de la UE (10 de marzo de 1995) y el Convenio Relativo a la Extradición entre los Estados miembros de la UE (27 de septiembre de 1996). En esta línea, el Parlamento Europeo adoptó, el 5 de septiembre de 2001, una resolución relativa al papel del bloque en la lucha contra el terrorismo invitando al Consejo a que adoptara una decisión marco tendiente a suprimir los procedimientos formales de extradición y aproximar las disposiciones legislativas, estableciendo normas sobre las penas en el ámbito del terrorismo, entre otras medidas penales (Villalba Fernández, 2007).

A partir de las medidas policiales y judiciales implementadas para profundizar en las acciones de cooperación relacionadas a la seguridad, se pueden identificar las siguientes generalidades: 1. Reforzar las normas a fin de impedir nuevas formas de terrorismo, 2. Intensificar los controles en las fronteras exteriores y de las armas de fuego, 3. Crear un organismo específico destinado a refrenar la propaganda terrorista en línea y 4. Armonizar las medidas legislativas en la materia.

Además, a partir del año 2007, Europol comenzó a elaborar informes de situación y tendencias del terrorismo. Estos estudios proporcionaN al público en general, a los agentes del orden y a los legisladores una visión del fenómeno del terrorismo en el año en cuestión, haciéndolo una de las piezas informativas más significativas de análisis estratégico.

Cabe mencionar que, dentro del bloque, la lucha contra el terrorismo tiene un seguimiento por parte de diversos grupos creados para tal fin. Entre estos grupos se destacan:

- El Grupo de Trabajo sobre los aspectos internacionales del terrorismo (COTER), en el que se analiza, discute e intercambia información sobre los asuntos de actualidad en el ámbito del terrorismo internacional, y se preparan los diálogos en materia anti-terrorista que la UE mantiene con terceros países.

- El Grupo de Trabajo sobre terrorismo (TWG), centrado en aspectos operacionales de política antiterrorista.

- El Grupo de Trabajo COMET de aplicación de medidas específicas de lucha contra el terrorismo, responsable de la monitorización de la lista antiterrorista de la UE. 
También existen varios organismos europeos con competencia en materia de lucha contra el terrorismo como el Si Cen (análisis de la amenaza), la ya mencionada Europol (Oficina Europea de Cooperación Policial), Eurojust (Cooperación Judicial Internacional y Coordinación de las investigaciones y procedimientos) y el grupo antiterrorista del Club de Berna que reúne los servicios de seguridad interna de 19 Estados.

Se puede destacar la implementación de la cláusula de solidaridad (artículo 222 del Tratado de Funcionamiento de la UE) y de la Cláusula de Defensa Mutua (recogida en el artículo 42.7 del Tratado de la UE), con el objetivo de reforzar las estrategias de seguridad y defensa colectiva de los Estados miembros

La cláusula de solidaridad establece la posibilidad de que la UE y los Estados miembros actúen conjuntamente para prevenir la amenaza terrorista en el territorio de un país de la UE o para prestar ayuda a otro país de la UE que sea víctima de una catástrofe natural o de origen humano. Uno de sus objetivos es lograr una estrategia antiterrorista común, aunque no completamente comunitarizada, capaz de movilizar los medios legales, policiales, judiciales, de información e inteligencia, incluso militares, para prevenir las actividades terroristas, $\mathrm{o}$, al menos, limitar su número y sus efectos dentro y fuera de la UE. Sin embargo, la cláusula de solidaridad no es una cláusula de asistencia, puesto que está limitada a unos supuestos concretos (terrorismo y catástrofes, en este caso), y no puede ser evocada en la mayor parte de los casos de agresión directa.

Es por esta razón, que el TUE en el Artículo 42.7 contempla la cláusula de Defensa mutua, la cual puede ser activada en caso de agresión directa a algún territorio de un Estado miembro. En concreto, el Artículo 42.7 establece que:

Si un Estado miembro es objeto de una agresión armada en su territorio, los demás Estados miembros le deberán ayuda y asistencia con todos los medios a su alcance, de conformidad con el artículo 51 de la Carta de las Naciones Unidas. Ello se entiende sin perjuicio del carácter específico de la política de seguridad y defensa de determinados Estados miembros. Los compromisos y la cooperación en este ámbito seguirán ajustándose a los compromisos adquiridos en el marco de la Organización del Tratado del Atlántico Norte, que seguirá siendo, para los Estados miembros que forman parte de la misma, el fundamento de su defensa colectiva y el organismo de ejecución de ésta (Tratado de la Unión Europea, 1992).

Es interesante resaltar que esta cooperación puede llevar a que los Estados miembros no sólo movilicen los instrumentos propios de las autoridades judiciales y de las fuerzas de seguridad, sino que aporten medios militares para: prevenir la amenaza terrorista, proteger las instituciones democráticas y a la población civil de posibles ataques terroristas y prestar asistencia a un Estado miembro en el territorio de éste, a petición de sus autoridades políticas, en caso de ataque terrorista.

\subsection{La renovación de la Estrategia de Seguridad Europea a partir de los atentados de 2015}


El año 2015 generó un antes y un después en la historia de la lucha contra el terrorismo en la región europea. La serie de atentados cometidos, llevaron a que los Estados miembros de la UE busquen aunar esfuerzos y tomar medidas más extremas para hacer frente a tan complejo fenómeno.

Si bien la responsabilidad principal de luchar contra el terrorismo y de garantizar la seguridad recae en cada uno de los Estados miembros, los atentados terroristas de los últimos años dejaron evidencia de que la seguridad es también una responsabilidad común en todo el bloque, debido a que la UE contribuye a proteger a sus ciudadanos ayudando a los Estados miembros. Así también lo manifestaba el Jefe de Gendarmería en la Embajada Francesa en Argentina Coronel Joel Molera, planteando que hoy en día no se puede luchar contra el terrorismo de manera aislada y que la cooperación tanto interna como externa en la UE, es una de las herramientas principales con las que cuentan los Estados para hacerlo.

En este sentido, en palabras del Coronel Joel Molera:

La UE, tras la serie de atentados perpetrados a partir de 2015, decidió incorporar nuevas medidas de seguridad en todo el bloque. Entre las medidas adoptadas se destacan la creación de un Centro para la lucha contra el terrorismo, un aumento de la cooperación respecto al control fronterizo, incremento en el presupuesto militar, mayor vigilancia y control a nivel regional ${ }^{10}$.

Como consecuencia de los atentados se acordó reforzar y acelerar una serie de medidas acordadas, que incluyen: a) Adopción de una Directiva para la lucha contra el terrorismo para la prevención de futuros atentados y hacer frente al problema de lobos solitarios; b) Refuerzo de los controles en las fronteras exteriores; c) Mejora del intercambio de información; d) Creación de un Centro Europeo de Lucha contra el Terrorismo (ECTC, por sus siglas en inglés); e) Refuerzo de la cooperación con terceros países (ligados al terrorismo), entre otras.

Por tal motivo, Europa decidió rediseñar su Estrategia Europea de Seguridad en el año 2016 bajo el nombre de Estrategia Global para la Política Exterior y de Seguridad de la UE, tras los atentados ocurridos a partir de 2015 en Estados como Francia, Bélgica y Alemania. Mediante la renovación de esta Estrategia, con su precedente en la de 2003, se buscó compartir información sobre el extremismo, redes terroristas y personas relacionadas o combatientes terroristas extranjeros; además del control y supresión de contenidos ilícitos en los medios de comunicación. Se definió al terrorismo y la delincuencia organizada como dos de las principales amenazas buscando trabajar en conjunto para evitar la financiación del terrorismo, así como intercambiar información. También, se subraya la necesidad de incrementar la lucha contra la radicalización y el reclutamiento de terroristas, así como aumentar el papel del diálogo intercultural en foros como el de la Alianza de Civilizaciones. Es importante destacar también que la UE aboga por reforzar los lazos con los países que más sufren este tipo de terrorismo, así como contribuir a fortalecer el buen gobierno y la seguridad de estos, de forma que se puedan adoptar medidas internas para evitar su

\footnotetext{
${ }^{10}$ Entrevista realizada por las autoras al Jefe de Gendarmería de la Embajada Francesa en Argentina, Coronel Joel Molera, el 11 de julio del 2019.
} 
propagación al resto del mundo. Del mismo modo, señala la necesidad de impulsar el intercambio de inteligencia entre los Estados miembros y sus socios para incrementar las capacidades para enfrentarse a las amenazas.

Otro punto relevante fue la activación de la cláusula de Defensa mutua especificada en el Artículo 42.7 del TUE la cual, como se mencionó con anterioridad, es una de las herramientas que obliga a prestar cualquier tipo de ayuda, incluida la militar, cuando un Estado sufra una agresión armada, terrorista o un grave perjuicio derivado de desastres naturales o humanos. Esta herramienta nunca había sido puesta en marcha hasta noviembre del año $2015^{11}$ cuando Francia, uno de los Estados miembros, invocó el artículo 42.7 con el objetivo de solicitar la ayuda y asistencia bilateral de los demás Estados miembros de la UE. Como respuesta, los ministros manifestaron unánimemente su pleno apoyo a Francia y su disposición a prestar toda la ayuda y asistencia necesarias. Este acontecimiento puso de manifiesto la necesidad urgente de desarrollar un verdadero compromiso a la hora de cooperar para combatir la amenaza terrorista.

Años más tarde, el 15 de marzo de 2017, la UE adoptó la Directiva sobre la lucha contra el terrorismo. La Directiva refuerza el marco legal para cubrir de manera más completa las conductas relacionadas con el terrorismo. Todos los Estados de la UE deben asegurarse de que tipifican como delito conductas como la formación y los viajes relacionados con el terrorismo, así como la financiación del mismo. Estas definiciones armonizadas sirven como punto de referencia para la cooperación y el intercambio de información entre las autoridades nacionales.

Antes de finalizar este análisis, es importante hacer mención a las medidas implementadas en los años más recientes, específicamente a partir de 2020, a raíz de los atentados terroristas cometidos en Francia, Alemania y Austria. A través de una Declaración Conjunta, en noviembre de 2020, los Estados miembros acordaron seguir intensificando su esfuerzo conjunto contra el terrorismo, sin comprometer los valores comunes del bloque, como la democracia, la justicia y la libertad de expresión (Consejo de la Unión Europea, 2020).

Previamente a esta declaración, en enero de 2020, fue creado el Centro de Asesoramiento de la UE para las Víctimas del Terrorismo, con el objetivo de asistir a los Estados miembros y a las organizaciones nacionales de apoyo a las víctimas, mediante directrices y actividades de formación. A su vez, la Comisión Europea adoptó una nueva Estrategia de Unión de Seguridad de la UE para el período 2020 a 2025, la cual presenta una serie de herramientas y medidas que desarrollarán durante esos 5 años.

Mirando hacia el futuro, en el 2022 se lanzará el nuevo esquema de exención de visado de Europa, denominado Sistema Europeo de Información y Autorización de Viajes (en adelante ETIAS, por sus siglas en inglés), el cual ayudará a combatir el terrorismo en Europa fortaleciendo las fronteras exteriores. Además de la implementación

11 Consejo de Asuntos Exteriores, 16 y 17 de noviembre de 2015 . Recuperado de: https://www.consilium.europa.eu/es/meetings/fac/2015/11/16-17 /

Esta obra se encuentra bajo la Licencia Creative Commons Reconocimiento - Compartir Igual (by-sa) 
de ETIAS, la UE está aplicando y tiene proyectado aplicar otras políticas para combatir el terrorismo. Las acciones de la UE para prevenir futuros ataques incluyen:

- Controles sistemáticos en las fronteras exteriores de Europa, tanto para los ciudadanos de la UE como para los visitantes de terceros países.

- Nuevo sistema de registro de entrada y salida para dejar constancia de los movimientos de ciudadanos no pertenecientes a la UE.

- Control temporal de las fronteras internas en ciertos países para evitar que los terroristas circulen libremente dentro de la UE y para evitar que personas de la UE viajen a zonas de conflicto para unirse a grupos terroristas yihadistas.

- Datos del registro de pasajeros como nombres, fechas de viaje, itinerario y método de pago compartido por las aerolíneas con las autoridades nacionales para prevenir, detectar, investigar y enjuiciar delitos terroristas, uso de identidad falsa y otros delitos graves.

- Fortalecimiento del Sistema de Información de Schengen (SIS) para introducir alertas sobre actividades relacionadas con el terrorismo, personas buscadas o desaparecidas y objetos perdidos o robados que la policía y los guardias fronterizos pueden consultar, así como un servicio de comparación biométrica para facilitar la identificación.

- Mayores competencias a Europol para avanzar en la lucha contra el terrorismo, además de apoyo en el intercambio de información entre las autoridades policiales nacionales y unidades especializadas como el Centro Europeo de Lucha Contra el Terrorismo.

- Reglas más estrictas para obstruir el acceso a los componentes necesarios para construir bombas caseras y otros explosivos que podrían usarse en ataques terroristas.

- Prevención de la radicalización y contraataque mediante la creación de una lista de vigilancia de predicadores de odio en toda la UE, segregando a los reclusos radicalizados en las cárceles, educando y trabajando en la inclusión social de las personas en riesgo.

- Y, por último, cooperación con Estados no pertenecientes a la UE para mejorar la seguridad exterior del bloque.

\section{Conclusiones}

El terrorismo representa un problema en el más estricto sentido del término; un problema que afecta la vida, la seguridad y el bienestar de muchas sociedades contemporáneas, pero también un problema teórico que ha suscitado fuertes debates 
conceptuales, metodológicos y hermenéuticos. De hecho, quien lo analice, se internará en un universo complejo, polémico y confuso, en el que los interrogantes exceden a las respuestas, las polémicas a los acuerdos y las confusiones a las certezas. En este contexto es que el estudio del terrorismo es casi una obligación ineludible: para los investigadores y académicos, que intentan explicarlo e interpretarlo a fin de encontrar mecanismos y procedimientos que contribuyan a frenar sus desafíos; para los gobiernos, que necesitan contar con herramientas que le permitan combatirlo en los diferentes ámbitos; y para los ciudadanos, que sufren directamente las consecuencias de los ataques.

A manera de conclusión, partiendo de la base de que el terrorismo se ha convertido en una de las principales amenazas para la región europea en la última década $\mathrm{y}$ de que esta situación ha generado la necesidad de dar una respuesta coordinada y apropiada, se sostiene que la UE ha sido capaz de evolucionar a través de los años en el desarrollo de una política integral antiterrorista, generando mecanismos de cooperación que han ayudado de forma esencial a las autoridades nacionales de sus Estados miembros a ser más eficientes y más eficaces para combatir el terrorismo. Por otro lado, es importante remarcar que las reticencias frente a la cooperación en este ámbito también han ido evolucionando hasta el punto de considerar hoy en día esa cooperación como esencial e indispensable para la lucha contra el terrorismo. Es así que se observa que la cooperación dentro del bloque europeo en la lucha contra el terrorismo es entendida como un objetivo de política en materia de seguridad y como un mecanismo para alcanzar la paz, la estabilidad regional y la de cada Estado miembro.

Los datos relevados instaron a reflexionar acerca de que los ataques terroristas a partir del año 2015, llevados a cabo por el EI en su mayoría, crearon un hito que alteró el sistema internacional no imaginado después de la caída de las torres gemelas el 11 de septiembre de 2001 y que continúa transformando la realidad internacional actual, obligando a la UE y sus Estados miembros a seguir reajustando el enfoque respecto a la seguridad y los mecanismos de cooperación. En definitiva, queda por ver cómo seguirá evolucionando este fenómeno, caracterizado por ser difuso y complejo, y cuál será la respuesta de la UE y sus Estados miembros frente al mismo, si continuarán apostando por los diferentes mecanismos de cooperación o si decidirán implementar otras alternativas para combatirlo.

\section{Referencias Bibliográficas}

AIXALÁ, A. (2007). La estrategia de la UE ante el terrorismo internacional y la defensa de los derechos y libertades. En Barbé, E. (coord.) Política exterior y Parlamento Europeo: hacia el equilibrio entre eficacia y democracia, Oficina del Parlamento Europeo-Institut Universitari d'Estudis Europeus, Barcelona. pp. 51-65.

ALBALADEJO, J. (2019). La política de la UE contra el terrorismo (I): del Tratado de Maastricht a la Estrategia de Seguridad Interior. Madrid: Real Instituto Elcano. Recuperado de:

http://www.realinstitutoelcano.org/wps/portal/rielcano_es/contenido?WCM_GLOBAL_C ONTEXT=/elcano/elcano_es/zonas_es/terrorismo+internacional/dt5-2019-albaladejo- 
politica-ue-terrorismo-tratado-maastricht-estrategia-seguridad-interior

BUSSO, A. (2003). El mundo después del 11 de septiembre. Revista del IRI. UNLP. pp. $127-141$.

CONSEJO DE LA UNIÓN EUROPEA (2020, 13 de noviembre). "Declaración conjunta de los Ministros de Interior de la UE sobre los recientes atentados terroristas en Europa". Comunicado de prensa. Recuperado de: https://www.consilium.europa.eu/es/press/pressreleases/2020/11/13/joint-statement-by-the-eu-home-affairs-ministers-on-the-recentterrorist-attacks-in-europe/\#

DIARIO OFICIAL DE LA UNIÓN EUROPEA. (2017, 31 de marzo). L 88. Recuperado de: https://eur-lex.europa.eu/legal-content/ES/TXT/?uri=OJ:L:2017:088:TOC

GRESH, A. (2015). Francia después de Charlie Hebdo. Estudios de Política Exterior, No45, marzo. Recuperado de. https://www.politicaexterior.com/articulos/afkarideas/francia-despues-de-charlie-hebdo

JORDÁN, J. (2013). Manual de estudios estratégicos y seguridad internacional. Madrid: Plaza y Valdés.

KEOHANE, R. (1988). Después de la Hegemonía. Cooperación y discordia en la política mundial. GEL. Buenos Aires.

KEOHANE, R. (1993). Instituciones internacionales y poder estatal. GEL. Buenos Aires.

MELLADO, N. (2002). "Los modelos de ALALC, ALADI y Mercosur", en Cátedra Internacional Andrés Bello/ Argentina (org.), Integración y Cooperación AtlánticoPacífico, CIDAM. UNR Editora. Rosario.

MORGENTHAU, H. (1986). Política entre las naciones. La lucha por el poder y la paz. GEL. Buenos Aires.

REINARES, F. (2011). Después de Osama Bin Laden: ¿cómo queda Al Qaeda y el terrorismo internacional? ARI, 83.

SPRINGLI, M. (2019). Características de las fuentes de cambio de la Política Exterior Francesa respecto del terrorismo yihadista durante el periodo 2015-2019. (Tesis de grado). Universidad Católica Argentina. Sede Paraná.

THIEUX, L. (2004). La Unión Europea frente al terrorismo global. Papeles N86, pp-95103. Recuperado de:

https://www.fuhem.es/media/cdv/file/biblioteca/PDF\%20Papeles/82-

86/THIEUX,\%20Laurence, \%20La\%20Uni\%C3\%B3n\%20Europea\%20frente\%20al\%20t errorismo\%20global,\%20Papeles\%2086.pdf 
TRATADO DE LA UNIÓN EUROPEA (1992). Recuperado de: https://eurlex.europa.eu/resource.html?uri=cellar:9e8d52e1-2c70-11e6-b497-

01aa75ed71a1.0023.01/DOC_2\&format=PDF

VELASCO TUDURI, S. (2013). Al Qaeda: origen, evolución y su presencia hoy en el mundo. Cuadernos de Estrategia, $\mathrm{N}^{\circ} 163$, pp. 155-184.

VILLALBA FERNÁNDEZ, A. (2007). "La Unión Europea en la lucha contra el terrorismo".Cuadernos de Estrategias. No $^{\circ} 135$, pp. 105-133.

\section{Sitios web consultados}

Web Oficial de la Unión Europea: https://europa.eu/

Web Oficial Comisión Europea: https://ec.europa.eu/

Web Oficial del Consejo Europeo y del Consejo de la Unión Europea: https://www.consilium.europa.eu/

Web Oficial Parlamento Europeo: https://www.europarl.europa.eu

Web Oficial de la Agencia de la Unión Europea para la Cooperación Judicial Penal (Eurojust): https://www.eurojust.europa.eu/

Web Oficial de la Agencia de la Unión Europea para la Cooperación Policial (Europol) https://www.europol.europa.eu/

Web Oficial de la Base Global de Terrorismo (por sus siglas en inglés, GTD): https://www.start.umd.edu/gtd/

Web Oficial del Ministerio para Europa y de Asuntos Exteriores de Francia: https://www.diplomatie.gouv.fr

\section{Cómo citar}

SPRINGLI, M. S. y CURVALE, P. (2021). La Cooperación Internacional en la lucha contra el terrorismo yihadista en el seno de la Unión Europea tras los atentados terroristas a partir del año 2015. Revista Integración y Cooperación Internacional, 33 (jul-dic), 16-34 